\title{
Philosophiques
}

\section{Deux conceptions de la neutralité de l'état}

\section{Pablo Da Silveira}

Volume 23, numéro 2, automne 1996

URI : https://id.erudit.org/iderudit/027394ar

DOI : https://doi.org/10.7202/027394ar

Aller au sommaire du numéro

Éditeur(s)

Société de philosophie du Québec

ISSN

0316-2923 (imprimé)

1492-1391 (numérique)

Découvrir la revue

\section{Citer cet article}

Da Silveira, P. (1996). Deux conceptions de la neutralité de l'état. Philosophiques, 23(2), 227-251. https://doi.org/10.7202/027394ar

\section{Résumé de l'article}

L'auteur part de la définition la plus intuitive du concept de neutralité pour présenter quatre thèses qui se sont révélées comme des acquis du débat. Ces thèses affirment : 1) que la neutralité est un concept politique et non pas moral ;2) qu'elle vise les justifications de l'action de l'État et non pas ses conséquences ; 3) qu'elle n'oblige pas nécessairement l'État à s'abstenir d'agir ; et 4) qu'elle est avant tout neutralité envers les individus et seulement de manière dérivée neutralité envers les conceptions du bien. Ensuite, l'auteur discute deux affirmations qui jouissent d'une popularité aussi forte que les précédentes mais qui sont beaucoup moins évidentes. La première est qu'il y a des liens profonds entre l'idée de neutralité et l'idée d'égalité. La deuxième est qu'il y a un rapport d'équivalence entre le concept de neutralité et celui d'anti-perfectionnisme. Mettre en question cette dernière affirmation implique une prise de distance par rapport à quelques argumentations bien connues en faveur de la neutralité, mais cette opération s'impose si on veut répondre aux critiques de ceux qui proposent l'abandon de cette exigence normative.
Ce document est protégé par la loi sur le droit d'auteur. L’utilisation des services d'Érudit (y compris la reproduction) est assujettie à sa politique d'utilisation que vous pouvez consulter en ligne.

https://apropos.erudit.org/fr/usagers/politique-dutilisation/ 
PHILOSOPHIgUES, VOL. XXIII, N ${ }^{\circ}$ 2, AUTOMNE 1996, P. 227-251

\title{
ARTICLES
}

\section{DEUX CONCEPTIONS DE LA NEUTRALITÉ DE L’ÉTAT}

\author{
PAR \\ PablO DA SIL VeIRA
}

RÉSUMÉ : L'auteur part de la définition la plus intuitive du concept de neutralité pour présenter quatre thèses qui se sont révélées comme des acquis du débat. Ces thèses affirment: 1) que la neutralité est un concept politique et non pas moral; 2) qu'elle vise les justifications de l'action de l'État et non pas ses conséquences ; 3) qu'elle n'oblige pas nécessairement l'État à s'abstenir d'agir; et 4) qu'elle est avant tout neutralité envers les individus et seulement de manière dérivée neutralité envers les conceptions du bien. Ensuite, l'auteur discute deux affirmations qui jouissent d'une popularité aussi forte que les précédentes mais qui sont beaucoup moins évidentes. La première est qu'il y a des liens profonds entre l'idée de neutralité et l'idée d'égalité. La deuxième est qu'il y a un rapport d'équivalence entre le concept de neutralité et celui d'anti-perfectionnisme. Mettre en question cette dernière affirmation implique une prise de distance par rapport à quelques argumentations bien connues en faveur de la neutralité, mais cette opération s'impose si on veut répondre aux critiques de ceux qui proposent l'abandon de cette exigence normative.

ABSTRACT: The article presents four theses that can be seen as firm outcomes of the contemporary debate on the principle of neutrality. These theses affirm that 1) neutrality is a political concept, not a moral one ;2) neutrality involves the justifications of the State's action, not its consequences;3) neutrality does not imply State's inactivity; and 4) State's neutrality is before anything neutrality between individuals and it is only afterwards between conceptions of the good. The second part of the article discusses two widely accepted but far more controversial statements. The first one says that there are deep links between neutrality and equality. The second one affirms that neutrality and anti-perfectionism are equivalent. To challenge the latter implies to reject some of the most popular cases for neutrality. However, this is needed if one wants to react to the great criticism coming from enemies of this principle. 
L'idée de neutralité de l'État, comprise d'une façon très large comme neutralité par rapport aux conceptions du bien choisies par les citoyens, est une des composantes les plus puissantes et les plus attirantes de la culture politique contemporaine. Elle est aussi au cœur de presque toutes les dëfenses significatives du libéralisme politique, entendant ce dernier comme une doctrine concernant le rôle de l'État dans le cadre d'une société pluraliste.

Malgré cette popularité - ou peut-être à cause d'elle - le concept de neutralité reste problématique. Il ne s'agit pas seulement du fait qu'il soit régulièrement soumis aux critiques de ceux qui rejettent le pluralisme politique, mais il pose des difficultés même à ceux qui sont prêts à s'engager à sa défense. John Rawls, par exemple, a renoncé à l'emploi du mot " neutralité " car il est à ses yeux soit trop confus, soit trop utopique ${ }^{1}$. Et même Bruce Ackerman, qui a fait du concept de neutralité une des clés de sa théorie de la justice, s'est demandé s'il n'avait pas commis une erreur quand il a fait ce choix ${ }^{2}$.

Ce malaise généralisé me semble tout à fait justifié. Nos idées sur la neutralité sont devenues trop aporétiques, trop ambiguës, trop incontrôlées. Néanmoins, je ne crois pas que ceci doive nous amener à l'abandon de cette exigence normative. Le concept de neutralité me semble toujours une composante décisive de toute théorie politique respectueuse du pluralisme, même si nos conceptions de la neutralité peuvent rencontrer des difficultés ${ }^{3}$.

Dans cet article, je voudrais me demander quelle est cette exigence minimale à laquelle nous ne pouvons pas renoncer si nous voulons respecter le fait du pluralisme : de quoi parlons-nous quand nous utilisons le mot "neutralité "dans la discussion politique? Jusqu'où peut-on l'employer avec une précision et une exactitude raisonnables ? Á partir de quand son usage devient imprécis ou controversé ? Je limiterai le cadre de mon analyse à la tradition

1. "The term "neutrality" is unfortunate; some of its connotations are highly misleading, while others suggest altogether impracticable principles ". John Rawls, Political Liberalism, New York, Columbia University Press, 1993. p. 191

2. "Perhaps I made a mistake, some time ago, in helping popularize the notion that something called Neutrality was at the heart of contemporary liberalism. But perhaps not. [...] Anyway, what's done is done ; all I can do now is try my best to clarify the confusion " (Bruce Ackerman, "Neutralities ", dans R. Douglas et al. (eds.) : Liberalism and the Good, New York, Routledge, 1990, p. 29).

3. Je prends appui sur une distinction proposee par H. L. A. Hart (The Concept of Law. Oxford, Clarendon Press 1961, p. 155 ss) et reprise par Rawls dix années plus tard (A Theory of Justice, Oxford, Oxford University Press 1971, p. 3-5) : un concept est ce qui est partagé par ceux qui discutent de façon intelligible sur une idée. Celle-ci reçoit des interprétations différentes incluant, entre autres choses, des critères d'application divergents. Chacune de ces interprétations est une conception du concept. "To illustrate : the concept of justice, applied to an institution. means, say, that the institution makes no arbitrary distinctions between persons in assigning basic rights and duties, and that its rules establish a proper balance between competing claims. Whereas a conception includes, besides this, principles and criteria for deciding which distinctions are arbitrary and when a balance between competing claims is proper. People can agree on the meaning of the concept of justice and still be at odds, since they affirm different principles and standards for deciding those matters. To develop a concept of justice into a conception of it is to elaborate these requisite principles and standards ". J. Rawls, Political Liberalism, p. $14 \mathrm{n}$. 
philosophique qui a le plus intensément discuté ces questions, à savoir, la philosophie politique anglo-saxonne postérieure à la publication de $A$ Theory of Justice de John Rawls. Je ne traiterai pas des sens que le mot peut avoir dans d'autres contextes de discussion et je ne ferai pas non plus de considérations historiques sur l'origine du débat ${ }^{4}$.

Ma dèmarche sera la suivante : je partirai de la définition la plus générale et la plus fréquente du concept de neutralité pour présenter ensuite quatre thèses qui qualifient ce concept et qui se sont révélées comme des acquis du débat. Ces thèses affirment : 1) que la neutralité est un concept politique et non pas moral ; 2) qu'elle vise les justifications de l'action de l'État et non pas ses conséquences ; 3) qu'elle est avant tout neutralité envers les individus et seulement de manière dérivée neutralité envers les conceptions du bien ; 4) qu'elle n'implique pas forcément l'inactivité ou le laissez-faire de l'Etat.

Ces affirmations résument l'essentiel de ce qui est devenu clair au cours de la discussion. À moins qu'on ne trouve des arguments jusqu'à présent inédits, elles doivent ètre considérées comme une base commune par tous ceux qui discutent de façon intelligible sur le sujet. Par contre, il y a d'autres aspects qui restent controversés. mais qui sont néanmoins décisifs pour la portée du concept. Parmi ces points conflictuels mais importants, je voudrais en discuter deux. D'abord je me demanderai quels sont les rapports entre la neutralité et le traitement égalitaire des individus. Je soutiendrai que la neutralité de l'État n'implique pas ce traitement égalitaire, mais que, par contre, un État qui veut faire preuve d'égal respect envers ses citoyens doit observer au moins une conception minimale de la neutralité. Ensuite je voudrais discuter la question de savoir si la neutralité entraîne nécessairement l'anti-perfectionnisme. Je soutiendrai que la réponse à cette question est négative.

\section{La neutralité comme concept politique ou comme concept moral ?}

Une définition assez classique de la neutralité présente celle-ci comme "le principe selon lequel l'État doit, par souci de justice, être neutre entre les différentes idées du bien ${ }^{5}$ ". Cette définition est trop générale et imprécise, mais elle a la vertu de souligner qu'il s'agit d'une exigence normative adressée à l'État et non pas d'un idéal moral s'appliquant à la vie privée des individus. Le sujet qui doit agir d'une façon neutre est l'Etat. Les individus (ou les associations d'individus) ne sont concernés par cet impératif que dans le cas où ils prennent part au débat préalable aux décisions qui seront prises par le pouvoir public.

Cette précision est importante parce qu'elle nous permet de faire la distinction entre la neutralité comme objet de la philosophie politique et d'autres sens possibles du mot. La neutralité politique est la neutralité par rapport au conflit sur la distribution des conditions

4. On peut trouver des références éclairantes sur ce dernier point dans Jeremy Waldron : "Legislation and Moral Neutrality ", dans R. Goodin \& A. Reeve, Liberal Neutrality. London, Routledge, 1989, p. 62 ss.

5. Brian Barry : "How Not to Defend Liberal Institutions ", dans B. Douglas et al. (eds.), Liberalism and the Good, New York, Routledge, 1989, p. 49. 
nècessaires à la mise en œuvre des différentes conceptions du bien présentes dans la société ${ }^{6}$. Elle ne nous concerne que dans la mesure où nous prenons part à ce conflit. Notre vie morale privée, par contre, reste au-delà de la portée de cette exigence ${ }^{7}$.

C'est seulement si on accepte ce présupposé que l'on peut comprendre, par exemple, l'équivalence proposée par Bruce Ackerman entre la neutralité et la "méthode de l'évitement " (method of avoidance). Aux yeux d'Ackerman, en effet, la neutralité exige que " sì vous et moi nous apprenons que nous sommes en désaccord sur l'une ou l'autre dimension de la vérité morale, nous ne devons pas chercher une valeur commune capable de neutraliser ce désaccord, ni chercher à le transposer dans un cadre de discussion censé ètre neutre, ou à le transcender en parlant sur la façon dont une créature extra-terrestre aurait pu le résoudre. Nous ne devons simplement rien dire à propos de notre désaccord, mais mettre les idéaux moraux qui nous séparent hors de l'agenda d'un État libéral ${ }^{8}$ ".

Quelle que soit notre opinion sur cette suggestion, une chose au moins devrait être claire : si nous appliquions cette méthode à notre vie morale privée, il est certain que le résultat serait une destruction plus au moins immédiate de toute sorte d'expérience morale. En effet, au moins dans les conditions imposées par la vie moderne, c'est avant tout au niveau de nos vies privées que nous formons nos opinions sur le bien, les confrontons avec celles des autres et essayons de définir un projet de vie plus ou moins cohérent avec nos conclusions. Or pour faire cela il faut que nos différences d'opinion sur ce qui donne de la valeur à la vie puissent être l'objet explicite d'une discussion.

Ackerman est-il un philosophe à ce point incompétent qu'il n'arrive mème pas à percevoir l'importance de cette condition pragmatique de notre vie morale ? Pas du tout. Il en est parfaitement conscient et il présente sa méthode justement comme une façon de fournir aux individus les conditions leur permettant de s'engager dans une telle recherche privée du bien. Tout simplement, il croit là tort, à mes yeux) que ces conditions ne seront assurees que si toute discussion à propos du bien est bannie du débat public.

\section{Neutralité des conséquences ou neutralité des justifications ?}

Une conception politique de la neutralité peut viser les conséquences de l'action de l'État ou bien ses justifications. Un principe de neutralité des conséquences instaure une distinction entre les buts qu'un État peut légitimement poursuivre et ceux qu'il ne peut poursuivre. Ainsi un tel principe peut affirmer que l'État ne

6. Charles Larmore, Patterns of Moral Complexity, Cambridge, Cambridge University Press, 1987, p. 74.

7. "Liberal democracies should be based on neutral principles - that is, on principles that promote the achievement of democratically defined social goals, without forcing dissenters to adopt these goals as their own". L. Becker \& W. Kymlicka, "Introduction to Symposium on Citizenship, Democracy, and Education ", Ethics 105, 3, 1995, 465.

8. Bruce Ackerman, "Why Dialogue?", The Joumal of Philosophy, 86/1, p. 16 .

9. Joseph Raz, The Morality of Freedom, Oxford, Clarendon Press, 1986, p. 107 ss. 
peut ni promouvoir ni faire obstacle à la réalisation d'une quelconque conception du bien, ou que l'État doit veiller à ce que ses politiques aient des conséquences égales pour toutes les conceptions du bien ou pour tous les individus, ou encore que l'État doit agir de façon à assurer un poids égal à toutes les conceptions du bien. La neutralité des justifications, quant à elle, interdit à l'État d'agir sur la base de certaines raisons et l'autorise à agir selon d'autres. Elle nous amènera à dire que l'État ne peut faire appel à aucune conception du bien particulière pour justifier ses actions, ou que l'État ne doit faire appel à aucune justification qui puisse être contestée, ou encore que l'État ne peut pas faire appel à des conceptions du bien incluant des jugements sur la valeur des individus ou des autres conceptions du bien. Cette énumération n'est pas exhaustive ${ }^{10}$.

Ces deux façons de concevoir la neutralité sont conceptuellement possibles a priori. Toutefois, le débat a apporté deux arguments décisifs à l'encontre de l'idée d'une neutralité des conséquences : le premier montre qu'elle n'est pas réalisable, le second montre qu'elle n'est pas désirable.

Le premier argument montre que la neutralité des conséquences est auto-contradictoire (et que par suite elle est impraticable), car tout essai de compenser des conséquences inégales entraînerait des effets aussi inégaux. En effet, toute intervention de l'État va nécessairement faciliter ou faire obstacle à la réalisation des différentes conceptions du bien. Cela semble évident dans le cas d'un État perfectionniste (engagé en faveur d'une conception spécifique du bien), mais la situation est la mème dans le cas d'un État qui renonce à toute valorisation d'une telle conception et méme dans le cas d'un Étatgendarme $^{11}$. Comme Charles Larmore l'a souligné, au moins ceux qui désirent vivre une vie de voleur auront des difficultés pour satisfaire leur préférence ${ }^{12}$.

La seule façon d'échapper à cette difficulté consisterait à installer un État absolument abstentionniste qui se refuserait à agir même à l'égard d'actes de violence privée. Dans ce cas, la probabilité de réaliser un programme de vie dépendrait de la capacité de chacun de défendre son projet et de trouver les moyens pour le réaliser. Or par cette voie on retournerait à un état de nature où la question de la neutralité perdrait toute pertinence. Nous aurions donc résolu les

10. Je ne voudrais pas martyriser le lecteur, mais il faut rappeler qu'on peut encore ètablir des distinctions au niveau des conséquences entre equal satisfaction et equal fulfilment, ou bien entre une neutralité positive et une neutralité négative (P. Jones, "The ideal of the neutral state ", dans Robert E. Goodin \& Andrew Reeve (eds.), Liberal Neutrality, p. 17 ss. On peut distinguer aussi entre une dimension objective et une dimension subjective de la neutralité (R. Arneson, "Neutrality and Utility ", Canadian Joumal of Philosophy 20/2,1990, p. 217). Et on peut appliquer cette distinction soit à la neutralité envers les individus, soit à la neutralité envers les conceptions du bien. Comme le but de ce paragraphe est de montrer l'impossibilité globale d'une neutralité des conséquences, je ne rentrerai pas dans ces détails.

11. Sur ce point voir R. Dworkin, "The Ethical Basis of Liberal Equality ". Dans University of Siena, International School of Economic Research (ed.), Ethics and Economics (2), Siena 1991, p. 44 et W. Kymlicka, Contemporary Political Philosophy, Oxford, Oxford University Press, 1990, 233 n. 4.

12. C. Larmore, Pattems of Moral Complexity, p. 43. 
problèmes posés par l'État neutre par la simple destruction de toute forme d'État.

Le deuxième argument à l'encontre de la neutralité quant aux conséquences affirme que même si elle était praticable, elle serait néanmoins indésirable. Cet argument ne vise donc pas les difficultés d'application de la neutralité des conséquences, mais il pose une objection normative à ses résultats.

Même si elle était applicable, en effet, la neutralité des conséquences serait d'abord incompatible avec la protection des libertés civiles et politiques, car le respect de ces garanties implique nécessairement des conséquences qui ne peuvent pas ètre les mêmes pour tous. Par exemple, certains individus auront des difficultés assez importantes pour développer la carrière de grand criminel à laquelle ils rêvent, et des groupes qui défendent une politique autoritaire devront admettre l'impossibilité d'emprisonner ceux qui ne partagent pas leurs opinions. Il n'est pas vrai que dans une société respectueuse des libertés toutes les préférences ont le mème poids. Cela veut dire que, soit on admet le principe de la neutralité des conséquences - et on ne fait donc pas obstacle aux projets de vie des criminels et des dictateurs par vocation - soit on admet la priorité de l'égal respect des libertés et on laisse donc tomber l'idée d'un État neutre quant aux conséquences de ses actions ${ }^{13}$.

En plus de cette incompatibilité avec l'égal respect des libertés, presque toutes les modalités imaginables d'une neutralité des conséquences entrent aussi en conflit avec le principe de responsabilité à l'égard de nos choix, c'est-à-dire avec le principe qui établit que personne n'a le droit de faire payer aux autres les coùts de la forme de vie que lui-mème a choisie ${ }^{14}$. En effet, notre sens de la justice nous dicte qu'un homme qui demande des ressources pour collectionner des voitures de sport et un homme qui demande des ressources pour faire face à un grave handicap physique ne doivent pas recevoir la même réponse de la part de l'État. Comme l'écrit Kymlicka, "ceux qui ont développé des goûts dispendieux sans prendre en compte ce à quoi ils peuvent raisonnablement aspirer ne peuvent pas exiger d'être subventionnés par les autres, et cela quelle que soit l'intensité de leurs désirs ${ }^{15}$ ". Or, si nous pratiquons la neutralité des conséquences nous pourrons justifier une réponse négative dans les deux cas ou bien nous pourrons justifier une égale satisfaction de chacun des deux désirs, mais nous ne pourrons pas justifier une réaction différenciée.

La neutralité des conséquences est donc en conflit avec notre sens de la justice. Et même si les appels à notre sensibilité morale n'ont pas la valeur d'une preuve, nous ne pouvons aller contre une

13. Sur cette discussion voir W. Kymlicka, "Liberal Individualism and Liberal Neutrality ", Ethics 99/4, 1989, p. $884:$ J. Rawls, A Theory of Justice, p. 250 et W. Sadurski, Moral Pluralism and Legal Neutrality. Dordrecht, Boston \& London, Kluwer Academic Publishers, 1990, p. 100.

14. Voir sur ce problème R. Dworkin, "What is Equality (2) : Equality of Resources.. Philosophy \& Public Affairs 10/4, 1981, p. 289 et J. Rawls, Political Liberalism, p. 34. Une conception de la neutralité des conséquences comme non-intervention échappe à cette deuxième partie de l'objection, mais elle est loin de pouvoir échapper à la première.

15. W. Kymlicka, "Liberal Individualism and Liberal Neutrality ", p. 885. 
intuition aussi ferme que si nous pouvons avancer des raisons de principe très puissantes. Or, le fait est que, au moins jusqu'à aujourd'hui, personne n'a été capable de faire des progrès dans cette direction, tandis que nous avons toujours des bonnes raisons pour croire qu'agir de façon juste envers les individus exige que chacun paye les coûts de ces choix.

Cet ensemble de difficultés nous montre que la neutralité des conséquences n'est pas praticable ni même désirable ${ }^{16}$. La neutralité de l'État ne peut être qu'une neutralité au niveau des justifications, et cela implique que les résultats de ses actions pourront toujours avoir des impacts différents tant sur les individus que sur leurs conceptions du bien ${ }^{17}$.

\section{La neutralité comme abstention ou comme guide pour l'action?}

La neutralité pose-t-elle toujours une limite à l'action de l'État ou peut-elle aussi exiger des actions positives ? La réponse à cette question reste ètroitement liée à l'ìmpossibilité d'une neutralíté des conséquences. En effet, si la seule neutralité possible est la neutralité au niveau des justifications, il n'y a d'autres limites à l'action de l'État que celles imposées par le besoin de foumir une justification qui soit effectivement neutre. Comme Larmore le souligne, "la neutralité politique consiste dans une contrainte relative aux facteurs qui peuvent ètre invoqués pour justifier une décision politique ", de sorte qu'elle "laisse en grande mesure indéterminés les buts que l'État devrait poursuivre. Bien sûr, certains buts (par exemple, l'établissement d'une religion d'État) ne sont pas acceptables car aucune décision neutre ne peut nous amener à les poursuivre. Mais tous les buts dont la poursuite peut ètre justifiée de façon neutre peuvent être ceux d'un État libéral ${ }^{18}$ ".

Le principe de neutralité n'exige l'abstention que par rapport aux actions qui ne peuvent pas ètre justifiées d'une façon neutre. Mais dans beaucoup d'autres situations, il peut exiger de l'État une action délibérée et mème des politiques assez complexes. Par exemple, un État neutre peut développer une politique de protection de l'environnement très ambitieuse en disant qu'il le fait justement parce qu'il est neutre : ce n'est qu'en protégeant les ressources naturelles que nous éviterons de faire des distinctions injustifiables entre les membres des générations présentes et les membres des générations futures. Cette politique restera compatible avec le respect de la neutralité méme si elle exige beaucoup d'actions délibérées de la part de l'État

16. Cette conclusion est partagée parmi d'autres par John Rawls ("The Priority of Right and Ideas of the Good ", Philosophy \& Public Affairs 17/4, 1988, p. 263). Bruce Ackerman ("Neutralities ". p. 39), Thomas Nagel (Equality and Partiality, Oxford, Oxford University Press, 1991 , p. 155) et Amy Gutmann ("Civil Education and Social Diversity", Ethics 105, 1995, p. 559).

17. Rawls trouve dans le rejet de la neutralité des conséquences une raison pour rejeter aussi l'utilisation du mot neutralité lui-même: "Neutrality of effect or influence political liberalism abandons as impracticable, and since this idea is strongly suggested by the term itself, this is a reason for avoiding it ". J. Rawls, Political Liberalism p. 194.

18. C. Larmore, Pattems of Moral Complexity, p. 44. 
et mème si elle fait obstacle à plusieurs programmes de vie spécifiques.

Un État neutre ne doit donc pas être nécessairement un État minimal ${ }^{19}$. Si quelqu'un voulait défendre cette idée, il devrait apporter la preuve empirique que la façon la plus efficace de satisfaire le principe de neutralité consiste à réduire le plus possible la sphère d'action de l'État ${ }^{20}$. Bien sûr, ceci ne veut pas dire qu'un État neutre doive ètre interventionniste. Mais cette combinaison n'a rien de contradictoire, comme le montre l'exemple bien connu de Bruce Ackerman $^{21}$. Cette possibilité de défendre une conception "interventionniste " de la neutralité permet de comprendre le fait que plusieurs philosophes qui sont favorables à la neutralité de l'État ne soient pas d'accord sur la manière dont un État neutre devrait agir.

\section{Neutralité envers les individus ou neutralité envers les conceptions du bien?}

Un État ayant fait un choix en faveur de la neutralité doit encore décider s'il va ètre neutre envers les individus ou bien envers les conceptions du bien choisies par ceux- $\mathrm{ci}^{22}$. Par " neutralité envers les individus "j'entends la neutralité envers des individus qui ont déja choisi une conception du bien particulière (mème s'ils peuvent la changer en tout moment). Par "neutralité envers les conceptions du bien "j'entends la neutralité envers la probabilité qu'une conception du bien (c'est-à-dire, une réponse spécifique à la question : "qu'estce que la vie bonne? ") soit choisie et mise en pratique plutôt qu'une autre. J'utiliserai les expressions " conceptions du bien " et "idéaux " comme des synonymes.

Un État qui se veut neutre envers les individus essayera soit de ne pas modifier les chances qu'ils ont de réaliser leur conception du bien, soit de les favoriser ou de les défavoriser en égale mesure, soit enfin de ne pas agir envers les individus en fonction d'une évaluation contestable de leurs motivations ou de leurs qualités intrinsèques. Dans les deux premiers cas, l'État essayera d'être neutre au niveau des conséquences de son action, mais on sait dejjà que cette possibilité n'est ni praticable ni mème désirable. Dans le dernier cas, l'État essayera d'être neutre au niveau des justifications, c'est-à-dire, au niveau des raisons qu'il considérera comme légitimes pour soutenir ses actions.

Un État qui se veut neutre par rapport aux idéaux essayera soit de ne pas favoriser ou défavoriser les chances qu'a chaque conception du bien d'être choisie et mise en pratique par les individus, soit d'affecter ces chances dans la mème mesure, soit de s'abstenir d'agir envers les différentes conceptions du bien en vertu d'une évaluation contestable de leurs excellences intrinsèques. Encore une fois, dans

19. De mème qu'un État minimal ne doit pas être forcément un État neutre : un Etat peut décider d'intervenir très peu sur la vie sociale, mais avec le but d'assurer la survie d'une conception du bien spécifique.

20. Sur ce probleme voir Cass Sunstein, "Neutrality in Constitutional Law (With Special Reference to Pornography, Abortion, and Surrogacyl ", Columbia Law Review 92/1, 1-52, 1992, p. 5 ss.

21. Bruce Ackerman, Social Justice in the Liberal State, New Haven, Conn.,Yale University Press, 1980, et The Future of Liberal Revolution, New Haven, Conn., Yale University Press, 1992.

22. J. Raz, The Morality of Freedom, p. 111-112. 
les deux premiers cas l'État essayera d'être neutre par rapport aux conséquences, tandis que dans le dernier cas il cherchera à ètre neutre au niveau des justifications de ses actions.

Une fois établie la distinction entre neutralité envers les individus et neutralité envers les idéaux, il faut se demander si l'État peut ètre neutre par rapport aux deux choses en même temps et, si cela n'est pas le cas, s'il est possible de trouver un critère de priorité.

Il est clair d'abord que, au niveau des conséquences, l'État ne peut pas ètre neutre à la fois par rapport aux individus et par rapport aux conceptions du bien. En effet, si l'État est neutre par rapport aux idéaux, il devra s'abstenir d'influer sur les chances qu'a chaque idéal d'être choisi et réalisé, ou bien il devra essayer d'égaliser ces chances. Mais qu'il fasse une chose ou l'autre, il affectera les chances qu'ont les individus de réaliser un de ces idéaux spécifiques. Par contre, si l'État ne veut pas altérer les chances de réussite des individus (ou s'il veut les égaliser) il modifiera la probabilité qu'un idéal spécifique soit choisi et réalisé. Mais cette incompatibilité n'a aucun effet sur la discussion parce que, comme l'on sait déjà, la neutralité des conséquences est en elle-même impraticable.

Les choses sont assez différentes dans le cas de la neutralité des justifications. En effet, un État qui s'interdit de prendre parti dans la discussion sur les conceptions du bien peut, au moins en principe, se réclamer neutre tant par rapport aux idéaux que par rapport aux individus. C'est par exemple la position d'Ackerman ${ }^{23}$ et on interprète aussi très souvent en ce sens l'anti-perfectionnisme rawlsien exprimé dans l'idée du voile d'ignorance ${ }^{24}$.

Mais il y a une différence décisive entre ces deux façons de comprendre la neutralité : la neutralité envers les individus bénéficie d'une certaine priorité par rapport à la neutralité envers les idéaux, dans le sens où l'État peut limiter le nombre des conceptions du bien en en appelant à la neutralité envers les individus, alors qu'il ne peut pas limiter la liberté des individus en en appelant à la neutralité envers les conceptions du bien ${ }^{25}$.

En effet, la neutralité envers les individus implique le respect de leur capacité de choisir une conception du bien, ainsi que le respect de tout choix qui résulte de son exercice. La neutralité envers les idéaux en découle nécessairement, car le fait de stimuler ou de faire obstacle à la réalisation d'une conception du bien spécifique implique une forme de pression sur cette liberté ${ }^{26}$. Néanmoins, cet égal respect envers les idéaux ne signifie pas que tout choix individuel ait le même droit à être réalisé. Une conception du bien qui nous amène à limiter la liberté de choix des autres sera toujours en conflit avec un État neutre envers les individus, et cela justement à cause de sa fidélité à cette exigence. L'égal respect des idéaux n'implique donc pas une latitude totale laissée à tous les programmes de vie imaginables mais une restriction imposée aux arguments qu'on peut utiliser pour leur faire obstacle. Un État neutre ne pourra pas

23. "Neutralities ", p. 37.

24. Voir, par exemple, P. Jones, "The ideal of the neutral state ", p. 9.

25. J. Rawls, "The Priority of Right and Ideas of the Good ", p. 253-256.

26. Peter De Marneffe, "Liberalism, Liberty, and Neutrality ", Philosophy \& Public Affairs 19/3, 1990, p. 253. 
s'opposer à la réalisation d'un idéal en vertu d'une évaluation contestable de sa valeur intrinsèque, mais il pourra le faire (bien plus il le devra) si sa realisation implique une atteinte aux droits des autres individus.

\section{Y-a-t-il des liens nécessaires entre la neutralité et l'égalité ?}

Jusqu'ici j'ai présenté quatre thèses sur le concept de neutralité qui, en l'état actuel de la discussion, peuvent ètre considérées comme relativement consensuelles et bien fondées. Elles affirment que : 1) la neutralité est un concept politique et non pas moral ; 2) la neutralité vise les justifications et non pas les conséquences de l'action de l'État ; 3) la neutralité n'oblige pas nécessairement l'État à s'abstenir d'agir ; 4) la neutralité est avant tout une neutralité envers les individus et seulement de manière dérivée neutralité envers les idéaux. Ces thèses peuvent étre considérées comme des acquis de la discussion. Elles mettent en évidence quatre traits essentiels de la neutralité qui sont reconnus tant par ceux qui acceptent la validité normative du concept que par ceux qui la rejettent. Cette approbation ou ce rejet est, au moins en partie, motivé par le fait que l'on reconnait ou non comme pertinents et viables ces quatre traits essentiels.

Dans les deux sections qui suivent je laisserai de cōté tout effort récapitulatif pour adopter une perspective plus polémique. Je voudrais discuter, en effet, deux affirmations qui jouissent d'une popularité aussi forte que les précédentes mais qui me semblent beaucoup moins évidentes. La première de ces affirmations est qu'il y a des liens profonds entre l'idée de neutralité et l'idée d'égalité. La deuxième est qu'il y a un rapport d'équivalence entre le concept de neutralité et celui d'anti-perfectionnisme. Mettre en question ces affirmations implique une prise de distance par rapport à quelques argumentations bien connues en faveur de la neutralité, mais il me semble que cette opération s'impose si on veut garder l'intelligibilité du concept.

Le premier problème que je viens de mentionner est celui de savoir s'il y a des implications profondes entre les concepts de neutralité et d'égalité. Cette idée bénéficie d'une présomption fort favorable, car elle est acceptée par deux des plus ardents défenseurs du principe de neutralité : Bruce Ackerman et Ronald Dworkin. Néanmoins, il me semble possible d'argumenter que s'il est vrai qu'on peut défendre à la fois ces deux idéaux, il n'est pas facile de trouver des liens de nécessité conceptuelle nous amenant de l'un à l'autre.

Ackerman et Dworkin ont rendu familière l'idée qu'un État qui veut respecter l'égalité de ses citoyens doit agir de façon neutre. Telle était la thèse d'Ackerman dans Social Justice and the Liberal State ${ }^{27}$ et telle était aussi la thèse de Dworkin dans son célèbre article "Liberalism "28. Certes, chacun de ces philosophes a défendu cette thèse à sa manière ${ }^{29}$, mais ils partagent l'idée que l'action neutre

27. Voir note 21 .

28. Dans Stuart Hampshire (ed.), Public and Private Morality, Cambridge, Cambridge University Press, 1978.

29. Pour des justifications plus récentes voir Bruce Ackerman, "Why Dialogue? ". The Joumal of Philosophy 86/1, 1989, p. 5-22 et Ronald 
(c'est-à-dire, l'action fondée sur des justifications neutres) est le type d'action caractéristique d'un État respectueux du principe d'égalité.

Toutefois le fait que deux philosophes importants soient d'accord sur une thèse n'est pas une preuve en faveur de sa vérité. Nous devons donc nous demander quelles sont les raisons qui peuvent plaider en faveur d'un rapport conceptuel fort entre neutralité et égalitè. Et avant de répondre à cette question il importe de lever deux ambiguités. D’abord, il faut préciser la signification du concept d'égalité lui-même, au moins de manière à pouvoir répondre à la question : égalité de quoi ? Ensuite, il faut percevoir que la question que nous nous posons peut ètre comprise de deux façons différentes, car elle pourrait viser à savoir si la neutralité implique l'égalité ou si par contre c'est l'égalité qui exige la neutralité.

Pour lever la première ambiguité, je ferais appel à la distinction rendue classique par Dworkin entre égale sollicitude et égal respect ${ }^{30}$. La première idẻe implique l'égal souci des intérêts des individus, qu'il soit compris dans le sens d'une égale distribution des ressources, des opportunités, du bien-être, etc. La deuxième idée implique l'égal respect de l'indépendance morale des individus (dans un sens de cette expression que je préciserai tout de suite), ce qui exige l'égal respect des libertés fondamentales.

La neutralité implique-t-elle l'égalité au sens de l'égale sollicitude ou au sens de l'égal respect? La première de ces possibilités peut ètre rapidement mise à l'écart. En effet, personne ne semble affirmer que la neutralité implique une forme quelconque d'égale sollicitude. En revanche, plusieurs philosophes soutiennent la thèse contraire. Lexemple le mieux connu à cet égard est celui des philosophes libertariens ${ }^{31}$, qui soutiennent un Etat neutre mais compatible avec des formes extrêmes d'inégalité tant au niveau des ressources et des chances qu'au niveau du bien-ètre ${ }^{32}$. Dans tous ces cas, l'État reste neutre au sens où il ne prend parti ni pour ni contre les différentes conceptions du bien qu'ont choisies les individus. Mais il reste aussi indifférent à l'égale sollicitude dworkinienne, dans le sens où il ne se soucie ni des besoins des individus ni des différences entre leurs niveaux de bien-être ou entre leurs dotations de ressources.

Le cas des libertariens montre que l'on peut dèfendre la neutralitè de l'État tout en restant indifférent à l'exigence d'égale sollicitude. Mais le principe d'égalité peut avoir, nous l'avons noté, un second sens, celui de l'égal respect. Ce second sens peut être lié au premier (comme chez Dworkin) ou en être indépendant (comme chez Nozick). Quoi qu'il en soit, on peut montrer qu'un État qui se veut neutre par

Dwokin, "What is Equality (4) : Political Equality ", San Francisco University Law Review 22/1,1989, p. 1-30.

30. Dworkin affirme qu'un Etat doit agir envers les individus " with concern, that is, as human beings who are capable of suffering and frustration, and with respect, that is, as human beings who are capable of forming and acting on intelligent conceptions of how their lives should be lived *. R. Dworkin, Taking Rights Seriously. London, Duckworth, 1977, p. 272.

31. Pour une caractérisation du libertarianisme voir Philippe Van Parijs. Qu'estce qu'une société juste ? Paris, Éd. du Seuil, 1991

32. Voir, par exemple, Robert Nozick. Anarchy, State, and Utopia, New York. Basic Books, 1974. 
rapport à la justification de ses actions peut rejeter aussi cette deuxième façon de comprendre l'égalité.

Je peux, en effet, défendre la neutralité de l'État pour des raisons qui n'ont rien à voir avec un égal respect envers les individus. Je peux le faire, par exemple, parce que je suis sceptique et, par conséquent, je ne crois en la possibilité d'aucune forme d'évaluation normative. Ou je peux le faire parce que je crois que dans ces conditions je jouirai d'une plus grande liberté relative, même si cela n'implique pas une égale liberté pour tous les membres de ma sociêté.

Si j'embrasse le principe de neutralité pour une raison de cette sorte, je peux soutenir par ailleurs que la fonction de l'État consiste à renforcer les opinions morales majoritaires, non pas bien sûr à cause de leur valeur intrinsèque mais pour des raisons d'utilité ou d'efficience. Je peux affirmer, par exemple, que l'État est autorisé à ignorer les droits des membres d'une petite minorité, non pas à cause du caractère plus au moins aberrant de leur conception du bien mais en raison d'un bénéfice collectif ${ }^{33}$. De cette façon je pourrais donner mon consentement à des décisions justifiées de manière neutre et qui aient en même temps l'effet d'écraser les minorités: "Imaginez que l'État neutre soit conçu non pas comme celui qui traite les conflits en utilisant des règles neutres, mais comme celui qui résout les conflits en utilisant des procédures neutres comme le vote. Dans ce cas il serait d'une énorme importance qu'une conception du bien soit soutenue par $55 \%$ plutôt que par le $5 \%$ de la population ${ }^{34}$ ".

La neutralité n'implique donc pas forcément l'égalité, ni dans le sens d'une égale sollicitude ni dans le sens d'un égal respect. Mais il nous reste encore à savoir sj on peut affirmer que l'égalité implique la neutralité, c'est-à-dire si un État qui se veut ègalitaire dans un des deux sens évoqués doit agir aussi de façon neutre.

Il semble clair qu'un État peut ètre égalitaire dans le sens d'une égale sollicitude envers les citoyens et en mème temps rejeter le principe de neutralité. En effet, un État peut assumer une théorie perfectionniste des besoins individuels et il peut essayer de les satisfaire d'une façon égalitaire. En vertu d'une telle théorie, il peut, par exemple, décider de distribuer équitablement des livres religieux ou des boissons alcoolisées. Un tel Êtat pose que les individus seront heureux s'il satisfait ces besoins et qu'ils seront frustrés s'il ne les satisfait pas, et cela quelles que soient les opinions exprimées par les individus concernés.

La question devient, par contre, plus intéressante s'il s'agit de l'exigence d'égal respect : un État qui veut pratiquer cette conception de l'égalité, doit-il ètre nécessairement un État neutre? Pour Ackerman et pour le " premier "Dworkin ${ }^{35}$, la réponse à cette question est affirmative. En effet, l'égal respect consiste dans l'égale

33. Eric Mack discute une telle conception de la neutralité en l'appelant Benthamite neutralism Voir son article : "Liberalism, Neutralism, and Rights * dans J. R. Pennock \& J. W. Chapman (eds.) : Religion, Morality, and the Law (Nomos XXX), New York \& Londres, New York University Press, 1988, p. 47.

34. Peter Jones, "The ideal of the neutral state", Dans Robert E. Goodin \& A. Reeve, Liberal Neutrality, p. 14.

35. C'est-à-dire le Dworkin antérieur aux Tanner Lectures de 1990. 
reconnaissance de la capacité qu'ont les individus de choisir et de mettre en pratique une conception spécifique du bien. La neutralité, de son côté, empêche l'État de s'engager dans tout jugement de valeur par rapport à cette question. Or, ajoutent Ackerman et Dworkin, il est évident que la capacité de choisir et de mettre en pratique une conception du bien ne peut ètre exercée dans des conditions d'égalité que si l'État respecte cette interdiction.

Ce raisonnement nous rappelle, certes, quelque chose d'important : si un État se veut soucieux de l'égal respect des individus, il doit respecter leur capacité de choisir et de mettre en pratique une conception spécifique du bien. Mais cela ne nous permet pas d'accepter l'ensemble de l'argument, car il faut encore montrer que le respect de cette capacité de choix exige de l'État une abstention totale par rapport à la question du bien. Ce présupposé est peut-être plausible, mais il n'est pas évident. Et pour le confirmer ou le rejeter, nous devons nous demander ce que l'État doit exactement respecter pour rendre possible l'exercice de la liberté de choix. Ce n'est qu'en répondant à cette dernière question que nous pourrons savoir avec précision quelles sont les limitations à imposer à la justification des actions d'un État neutre.

J'essayerai donc de défendre ici la thèse suivante: un État pratiquant l'égal respect envers les individus est un État qui respecte leur indépendance morale. Celle-ci est la condition nécessaire et suffisante pour pouvoir choisir entre des différentes conceptions du bien, méme si elle n'arrive pas à nous assurer la pleine réalisation de la conception de notre choix. Pour préciser la portée de ce nouveau concept je procéderai d'abord par la négative, en le détachant de deux idees qui lui sont proches mais qui restent tout de mème différentes. Ensuite je proposerai une formulation positive. Enfin, je discuterai la question de savoir si un État respectueux de l'indépendance morale des individus doit effectivement s'abstenir de tout jugement de valeur, c'est-à-dire s'il doit rester aveugle par rapport à la question du bien.

\section{Indépendance morale}

L'indépendance morale est une forme d'autonomie morale individuelle mais qui se distingue de deux autres formes d'autonomie.

Elle est d'abord différente de l'idée kantienne d'autonomie comme caractère distinctif de notre nature morale. Cette idée, en effet, implique une théorie "profonde "sur notre statut d'agent moral qui peut faire l'objet d'un désaccord raisonnable. Il y a des arguments très puissants pour la soutenir mais il y en a aussi d'autres également respectables pour la mettre en question. Ce fait la rend inadéquate comme base d'une argumentation politique susceptible d'ètre partagée par des citoyens qui défendent des théories morales rivales. Bien sür, les théories "profondes" de cette sorte peuvent jouer un rôle important (voire central) dans notre vie morale personnelle et elles peuvent aussi ètre défendues dans un débat public. Mais si on essaye de les utiliser comme soutien d'un accord politique de base, on tombe dans ce que Charles Larmore a appelé une "perspective expressiviste", c'est-à-dire une perspective qui 
essaye de tirer des conclusions politiques partageables à partir d'une théorie extra-politique, controversée et difficile à contrōler ${ }^{36}$.

Je réserverai l'expression "autonomie morale " pour faire référence à l'idée kantienne d'autonomie et, en suivant une suggestion de Raz, j'utiliserai l'expression " autonomie personnelle " pour me référer à un deuxième sens du mot qui ne jouera, lui non plus, aucun rôle dans mon argumentation.

L'expression " autonomie personnelle " ne renvoie pas à une thèse sur notre nature morale mais à un idéal de vie. Dans les mots de Raz, "l'idéal de l'autonomie personnelle est la vision des gens contrôlant, jusqu'à un certain degré, leur propre destin, lui donnant forme par des décisions successives tout au long de leurs vies ${ }^{37}$ ". On peut suivre l'idéal d'autonomie personnelle parce qu'on partage la théorie kantienne de lautonomie morale mais on peut aussi le faire sans défendre aucune théorie de cette sorte. En tout cas, le fait essentiel est que l'on se conçoit comme maitre de sa propre vie. Comprise dans ce sens spécifique, "l'autonomie est un idéal d'autocréation $^{38}$ ". Elle reste compatible avec d'autres idéaux complémentaires mais différents, comme celui de doter sa propre vie d'unité et de cohérence, ou celui de l'auto-réalisation. Mais son noyau est l'idée d'une vie dans laquelle chaque engagement et chaque attachement a été (ou peut ètre) l'objet d'une décision délibérée ${ }^{39}$.

De très bons arguments plaident en faveur de cet idéal. Toutefois des arguments tout aussi valables peuvent plaider en faveur d'autres idéaux de vie, par exemple en faveur d'une vie en harmonie avec les attachements sociaux et culturels constitutifs de notre identite ${ }^{40}$. Lidéal d'autonomie personnelle peut être controversé. Il ne peut donc servir de fondement à un accord politique sous peine de tomber dans l'expressivisme.

En revanche, le concept d'indépendance morale ne risque pas de tomber dans ce piège. Il ne suppose, en effet, que la simple reconnaissance du fait que notre bien-être dêpend, au moins en partie, de la possibilité de mener nos vies from the inside, c'est-àdire de la possibilité de mener nos vies en fonction de nos propres convictions éthiques ${ }^{41}$. Lidée intuitive sous-jacente à cette affirmation est que, au delà de nos différences et de nos conceptions divergentes à propos du bien, tous les individus reconnaissent que le fait de mener leur vie comme ils l'ont choisi est une composante essentielle de leur bien-être.

Cette affirmation ne doit pas être interprétée comme une variante de l'idéal de l'autonomie personnelle. Celui-ci, en effet, n'est qu'une des réponses possibles à la question : "comment est-ce que je veux

36. C. Lamore, Pattems of Moral Complexity, p. 76 ss et p. 118 ss.

37. J. Raz, The Morality of Freedom, p. 369

38. Loc. cit., p. 370.

39. Pour une défense de cette sorte d'autonomie voir Stephen Macedo, "Charting Liberal Virtues ", dans J. W. Chapman \& W. A. Galston, Virtue (Nomos XXXIV), New York \& London, New York University Press, 1992, p. 214-218.

40. Voir, par exemple, Charles Taylor, "Atomism ", dans Philosophical Papers, vol. II, Cambridge, Cambridge University Press, 1985, p. 187 ss. et Alasdair MacIntyre, "Is Patriotism a Virtue?", The Lindley Lecture. Kansas, Kansas University Press, 1984.

41. W. Kymlicka, Contemporary Political Philosophy, p. 204. 
vivre ? ". Or, je peux répondre à cette question en disant que je veux mener une vie que je maitrise absolument, où tout engagement est le résultat d'une décision explicite, mais je peux aussi y répondre en choisissant une vie fortement ancrée dans des attachements constitutifs, par exemple de type national ou religieux. Le fait important est que dans les deux cas je me sentirai fort lésé si je ne peux pas mener le genre de vie que j'ai choisi. Je me sentirai fortement lésé si une société intolérante m'interdisait de mener ma vie conformément au principe du libre-examen, mais je me sentirai aussi lésé si je ne peux pas vivre une vie vouée à mes attachements constitutifs (parce que ma religion est interdite ou qu'elle a disparu de la société dans laquelle je vis). Le mécontentement qui est commun aux deux cas manifeste le souci commun de l'indépendance morale, tandis que les deux réponses opposées reflètent des jugements différents sur la valeur de l'autonomie personnelle.

L'idée d'indépendance morale ne présuppose aucune thèse anthropologique ni aucune théorie profonde et controversée concernant notre vie morale. Elle se limite à affirmer que le désir de choisir le genre de vie que nous voulons vivre fait partie de nos intérèts les plus essentiels, c'est-à-dire de ces préférences qui sont à la base de toutes nos préférences.

Cette affirmation ne prétend pas ètre valable pour toutes les sociétés et pour toutes les époques. Toutefois ceci ne signifie pas que cette thèse serait un pur constat factuel, affirmant seulement que l'idée d'indépendance morale est valable là où elle est reconnue comme valable et tant qu'elle est reconnue comme valable. Tout au contraire, on peut attribuer à cette thèse une force normative dès lors que l'on peut montrer que, au moins dans nos sociétés, elle est inhérente à l'interprétation que nous avons de notre vie morale et de nos institutions ${ }^{42}$.

Je ne me prononcerai pas ici sur les chances d'une telle argumentation. Je veux dire tout simplement que ce principe d'autonomie effective est la chose que nous visons à protéger quand nous essayons de protéger la liberté de choix des individus. Elle est. en effet, la condition minimale qui nous permet d'exercer notre liberté de choisir une conception spécifique du bien et de la mettre en pratique. S'il est vrai donc qu'un État soucieux de l'égal respect des individus est un État respectueux de leur liberté de choix, alors il est aussi vrai qu'un tel État doit chercher à assurer les conditions de leur indèpendance morale. Ceci est la base du rejet de l'État paternaliste, c'est-à-dire, d'un État cherchant à gouverner la vie des individus selon un idéal perfectionniste, et de toute intervention qui, par quelque moyen, essaie d'imposer aux individus une réponse plus au moins restrictive à la question : "Guel genre de vie est-ce que je veux vivre?".

Or, si on accepte qu'un État soucieux de l'égal respect est un État soucieux de l'indépendance morale des individus, doit-on en conclure qu'un État soucieux de l'égal respect doit ètre neutre ? Cela dépend du sens que nous donnons au mot "neutralité ". Si par "État

42. Derrière cette argumentation il y a l'idée d' interprétation constructive ", proposée par Ronald Dworkin dans Law's Empire, Cambridge, Harvard University Press, 1986. 
neutre " on comprend un État s'abstenant de toute intervention fondée sur des idées du bien (comme Ackerman et au moins le "premier " Dworkin le font), tout dépend de savoir si un État peut rejeter cette exigence tout en respectant néanmoins l'indépendance morale des individus. Si c'est le cas, la réponse à la question est négative. Si ce n'est pas le cas, la réponse sera affirmative. Si par contre, on trouve la façon de défendre une conception de la neutralité de l'État qui, tout en respectant l'autonomie effective des individus, n'implique pas cette interdiction extrēme, la réponse sera toujours positive.

\section{La neutralité implique-t-elle l'anti-perfectionnisme ?}

Jusqu'à présent j'ai défini la neutralité par le refus de justifier les actions de l'État en invoquant un jugement de valeur sur les individus ou sur les conceptions du bien choisies par eux. Mais cette formule contient une ambiguité qu'il faut maintenant lever. En effet, elle peut vouloir dire que cette interdiction oblige l'État à rester aveugle par rapport à tout jugement de valeur, ou bien elle peut vouloir dire qu'elle ne l'oblige à rester insensible que par rapport à certains de ces jugements. Par "jugements de valeur "je comprends ici des jugements sur la valeur intrinsèque des individus, des conceptions du bien ou des biens particuliers. Une chose a une valeur intrinsèque quand nous avons des raisons pour la choisir pour elle-méme et non pas en fonction de son utilité pour atteindre un autre objet de choix.

Beaucoup de philosophes optent pour la première interprétation : l'exigence de neutralité implique tout simplement que l'État ne peut faire appel à aucun jugement de valeur dans aucun domaine. Ce n'est qu'à cette condition qu'il ne fera pas de différences ni entre les individus ni entre les conceptions du bien ${ }^{43}$. Or dans cette section je voudrais suggérer que cette affirmation ne vise pas le concept de neutralité lui-même mais une conception de ce concept, à savoir, la conception anti-perfectionniste de la neutralitét ${ }^{44}$. Cela veut dire qu'il est possible de proposer une autre interprétation du concept qui avance qu'un État peut être neutre sans pour autant s'abstenir de faire appel à tout jugement de valeur dans la justification de ses actes.

Pour mieux préciser les choses, je présenterai ici deux conceptions alternatives du concept de neutralité. Je suggère : i) qu'un État n'est neutre que s'il met en pratique une de ces conceptions; et ii) que toute formulation du concept de neutralité peut ètre finalement réduite à l'une ou l'autre de ces conceptions. Je resterai au niveau de l'analyse conceptuelle et je ne discuterai donc pas les conséquences pratiques de chacune de ces alternatives.

43. Voir par exemple Bruce Ackerman, "Political Liberalisms ", The Journal of Philosophy 91/7, 1994, p. 373 ; R. Dworkin, A Matter of Principle, p. 19l, C. Larmore, Pattems of Moral Complexity, p. 43 et R. Nozick, Anarchy, State and Utopia, p. 33.

44. Voir note 3 . Cette section discute deux conceptions du concept de neutralité, qui a été présenté dans les sections précédentes. 


\section{La conception an ti-p erfectio nnis te}

La neutralité peut d'abord ètre comprise comme la négation de la pertinence de tout jugement de valeur dans la justification des actions de l'État. Le concept de neutralité implique, en effet, que ètant donnés deux groupes de personnes $A$ et $B$, l'Etat ne peut pas favoriser le groupe $A$ (et par conséquent défavoriser le groupe $B$ ) en disant que les membres du groupe $A$ possèdent une certaine excellence $x$ (leur race, leur patriotisme, leur richesse, etc.) qui leur est intrinsèque, ou en disant que l'idéal défendu par les membres du groupe $A$ possède une certaine excellence $y$ (sa religiosité, sa ferveur nationale, etc.) qui lui est intrinsèque. Dans une formule que Bruce Ackerman a rendu célèbre : " aucune raison n'est une bonne raison si elle requiert du détenteur du pouvoir d'affirmer: (a) que sa conception du bien est meilleure que celle affirmée par n'importe lequel de ses concitoyens, ou (b) que, indépendamment de la conception du bien qu'il défend, il est intrinsèquement supérieur à un ou à plusieurs de ses concitoyen ${ }^{45}$ ".

Cette exigence, qui exprime le cœur mème du concept de neutralité, oblige l'État à rester aveugle à tout jugement de valeur intrinsèque portant sur les individus ou sur les conceptions du bien choisies par les individus. Or - ajoute la conception anti-perfectionniste de la neutralité - elle oblige aussi l'État, quoique indirectement, à rester aussi aveugle à la valeur intrinsèque de tout bien particulier et de toute pratique particulière. En effet, ces biens et ces pratiques n'ont de la valeur pour nous qu'à la lumière d'une conception du bien spécifique. Dès lors, protéger certains biens ou certaines pratiques, ou même contribuer à les rendre plus disponibles, mènera nécessairement (au moins dans un contexte de rareté de ressources) à favoriser certaines conceptions du bien et à en défavoriser d'autres. Étant données une conception du bien $M$ qui valorise les biens ou les pratiques particuliers $a, b$ et $c$, et une conception du bien $N$ qui valorise les biens ou les pratiques particuliers $d$, e et $f$, si l'État protège ou prête son soutien aux biens particuliers $a$ et $c$, la conception du bien $M$ sera cæteris paribus plus facile à réaliser que la conception du bien $N$.

Dès que nous avons des raisons pour rejeter la neutralité des conséquences, rien n'empéche l'État de justifier des décisions de cette sorte par des raisons purement anti-perfectionnistes. Par exemple, il peut mener une politique d'alphabetisation (c'est-à-dire, de soutien à la pratique de la lecture) non pas parce que celle-ci serait une pratique précieuse en elle-mème, mais parce qu'elle est une condition pour l'exercice des droits du citoyen. Par contre, l'État doit toujours s'abstenir de toute discrimination entre des biens et des pratiques impliquant une évaluation de mérites intrinsèques. Par exemple, il ne peut pas justifier une politique d'alphabétisation en affirmant que lire est intrinsèquement meilleur que regarder la télévision. S'il ne respecte pas cette interdiction, l'État agit différemment envers les différentes conceptions du bien et cela pour des raisons qui ne peuvent pas ètre acceptées par l'ensemble des citoyens.

45. B. Ackerman, Social Justice in the Liberal State, p. 11. 
J'utiliserai le terme anti-perfectionnisme pour me référer à cette conception. Il s'agit d'une conception forte de la neutralité qui établit des limites très précises à la justification des actions de l'État et aux arguments qui peuvent ètre utilisés dans la discussion préalable à ces décisions. Elle implique que l'on ne peut dans cette discussion faire appel à l'idée d'excellence intrinsèque, et cela au nom de l'égal respect des citoyens.

L'anti-perfectionnisme ne doit pas ètre confondu avec le scepticisme moral. Le scepticisme est une doctrine concernant la nature du raisonnement moral qui soutient soit qu'il n'y a pas d'arguments moraux meilleurs que d'autres, soit que, mème s'il y en a, nous ne serons jamais capables de les identifier. Lanti-perfectionnisme, par contre, ne nie pas le fait que je puisse raisonnablement reconnaître des valeurs intrinsèques au niveau de ma vie morale personnelle. Mais il affirme que ce fait n'a aucune place dans le débat politique.

L'anti-perfectionnisme n'implique pas non plus le rejet de toute modalité collective de recherche du bien. Il affirme plus simplement que "les perfections humaines doivent ètre poursuivies sans sortir des limites du principe de libre association ${ }^{46}$ ". Les individus peuvent s'associer de façon volontaire dans des organisations culturelles, des églises, des associations professionnelles ou des universités. Mais ils ne peuvent pas exiger la satisfaction de leurs préférences par la voie d'une action de l'État. Les biens recherchés par les associations volontaires ne peuvent pas devenir des buts de l'action politique.

\section{La conception perfection nis te modeste de la neutralité}

La deuxième conception de la neutralité affirme également qu'un Etat neutre doit ètre aveugle vis-à-vis des jugements de valeur sur les individus et sur leurs idéaux, mais elle nie que cela l'oblige à rester aveugle à tout jugement de valeur. Du point de vue conceptuel, rien n'empêche qu'un État respectueux de la neutralité puisse ètre sensible à certains jugements sur la valeur des biens particuliers ${ }^{47}$ et qu'il puisse se servir de ces jugements dans la justification de ses actions.

J'utiliserai l'expression perfectionnisme modeste pour me référer à cette deuxième conception de la neutralité. Elle est perfectionniste dans le sens où elle accorde une certaine place aux jugements de valeur dans la discussion publique, mais elle reste modeste dans le sens où elle ne défend aucun standard de valeur indépendant du jugement des individus.

La conception perfectionniste modeste est fondée sur une critique du point de vue anti-perfectionniste. Celui-ci affirme, on l'a vu, qu'un État ne peut ètre neutre envers les individus et envers les conceptions du bien que s'il est aveugle à tout jugement de valeur.

46. J. Rawls, A Theory of Justice, p. 328.

47. Dorénavant, et pour raisons de simplicité, j'utiliserai le terme "biens " pour faire référence à l'ensemble des biens et des pratiques qui peuvent ètre l'objet de nos choix. La seule difference consiste lcomme on le sait depuis Aristotel dans le fait que les biens peuvent ètre détachés des actes qu'il faut accomplir pour les produire, tandis que cela n'est pas possible dans le cas des pratiques. 
On ne peut satisfaire la première condition que si on satisfait aussi la deuxième.

Or, dit le perfectionniste modeste, ce raisonnement est un non sequitur à moins qu'on accepte deux présupposés. Le premier est que notre intérèt fondamental consiste à réaliser la conception du bien que nous avons librement choisie. Le second est que toute conception du bien est un schéma déterminé de buts finaux, c'est-à-dire un schéma structuré de telle sorte que:a) étant donné n'importe quel but particulier, nous soyons toujours capables de savoir s'il fait partie ou non de notre conception du bien et avec quel degré d'importance ; et ii) étant donné n'importe quel bien particulier, nous soyons toujours capables de savoir quelle est la place qu'il occupe dans l'ordre de nos préférences en fonction de sa capacité de satisfaire les buts que nous nous sommes donnés ${ }^{48}$. Si ces présupposés sont exacts, alors il est vrai que le soutien apporté à n'importe quel bien particulier aura toujours pour effet de bénéficier à certaines conceptions du bien au détriment de toutes les autres. Ce fait suffit pour interdire à un État neutre de s'engager dans une telle action pour des raisons autres que des arguments purement antiperfectionnistes.

Toutefois ces deux présupposés sont très difficiles à admettre, car ils sont en conflit avec quelques données cruciales de notre expérience morale.

Tout d'abord, il est un fait que nous ne semblons pas seulement intéressés à réaliser notre conception du bien mais aussi à vérifier sa valeur. Nous savons que nous pouvons nous tromper dans nos choix. Nous ne visons donc pas à réaliser n'importe quelle conception du bien, mais à choisir la bonne conception du bien (une idée critique toujours indéterminée ${ }^{49}$. L'analyse de notre expérience morale individuelle, de méme que l'analyse du langage moral et des récits littéraires, témoignent de l'importance de ce phénomène: nous ne concevons pas notre bien comme la réalisation de la conception du bien que nous avons librement choisie mais comme la libre réalisation de la bonne conception du bien ${ }^{50}$. La liberté de choix n'est pas pour nous une fin ultime mais une condition pour mener cette recherche. Elle est importante à nos yeux parce que la quête de la meilleure façon de vivre nous semble importante ${ }^{51}$. Si nous

48. On trouve une défense explicite de ces deux présupposées (quoique plus nuancée que dans le passê) dans $J$. Rawls 1993, p. 311 ss. L'expression " conceptions du bien déterminées " lui appartient : "Citizens (are) regarded as having at any given time a determinate conception of the good, that is, a conception specified by certain definite final ends, attachements, and loyalties... *. Political Liberalism, p. 74, voir aussi p. 19-20 et p. 108.

49. Je suis ici les idêes de W. Kymlicka dans Liberalism, Community, and Culture, (Oxford. Clarendon Press, 1989, p. Il ss) même si, par des raisons que je ne peux pas discuter maintenant, il reste un défenseur du point de vue anti-perfectionniste.

50. Ceci n'implique pas qu'il y ait une conception du bien qui soit la meilleure pour tous les individus. Le bien de chacun peut être différent du bien des autres. En tout cas, mon argumentation n'exige pas qu'on prenne une position définitive sur ce point.

51. Cela n'empéche pas que quelqu'un puisse ériger la liberté de choix en but final. Mais le fait mème de devoir expliquer que la liberté de choix est pour lui un bien en soi implique qu'il comprend la différence entre sa valeur instrumentale (qui est générale) et la valeur intrinsèque qu'il lui assigne. 
n'acceptons pas ce présupposé, une bonne partie de nos actes et de notre langage moral devient incomprëhensible à nos propres yeux.

Ensuite, il est un fait que les rapports entre nos conceptions du bien et les biens particuliers que nous trouvons dans le monde sont plus complexes que le deuxième présupposé le suggère. D'un côté, il semblerait que nous avons des opinions sur des biens spécifiques qui sont relativement indépendantes de tout engagement avec une conception du bien déterminée. Le fait qu'une conception spécifique soit incompatible avec la jouissance d'un de ces biens est à nos yeux une raison pour l'abandonner ${ }^{52}$. Un individu peut abandonner une vie centrée sur le profit s'il acquiert la conviction que cette vie l'empecche de jouir d'une vie familiale chaleureuse ou d'avoir accès aux biens de la culture. Un autre peut abandonner une vie centrée sur la recherche philosophique s'il s'aperçoit que cela l'empèche d'accumuler des richesses. D'un autre côté, et pour compliquer encore les choses, il semblerait que ce qui compte comme un bien dépend, au moins en partie, de la conception du bien que nous avons choisie. Par exemple, pour beaucoup de personnes la beauté du paysage n'est pas du tout un bien. Leur conception du bien y est tout simplement aveugle. Pour d'autres, par contre, cette insensibilité peut être une raison de renoncer à une conception du bien. Encore une fois, nous ne nous limitons pas à choisir des biens particuliers en fonction de notre conception du bien mais nous faisons des opérations beaucoup plus complexes.

Les philosophes anti-perfectionnistes sont normalement prêts à admettre ce type de remarque, mais ils affirment qu'on peut quand méme procéder à certains simplifications méthodologiques pour des raisons d'économie théorique ${ }^{53}$. Le perfectionniste modeste, par contre, affirme que ces données de notre expérience morale ont une importance capitale car elles permettent d'affirmer que les biens qui comptent pour les individus ne sont pas seulement ceux qui servent comme des moyens à la réalisation de leur conception du bien (ou de n'importe quelle conception du bien) mais aussi ceux dont la disponibilité permet de faire des choix plus riches et plus réflexifs entre des différentes conceptions du bien. Par exemple, un patrimoine urbain plus riche est préférable à un patrimoine urbain plus pauvre, car cela offre - au moins dans certaines conditions culturelles - un contexte de choix plus varié. De même, la transmission d'une langue nationale ou de certaines traditions distinctives peut rendre plus intelligible et plus riche aux yeux des individus le contexte social et culturel dans lequel ils doivent faire leurs choix moraux $^{54}$.

Appelons " biens partagés" ces biens ayant la capacité de rendre plus riche notre contexte de choix. Certes, les membres d'une société

52. Charles Taylor appelle "hypergoods" les biens qui nous permettent d'evaluer d'autres biens (Sources of the Self. The Making of the Modern Identity, Cambridge, Cambridge University Press, 1989, p. 63). Mais je parle ici de ces biens dont la compatibilité ou l'incompatibilité nous permet d'évaluer des conceptions du bien.

53. Voir, par exemple, J. Rawls, Political Liberalism, p. 311.

54. C. Taylor, "The Politics of Recognition", dans C. Taylor et al., Multiculturalism and * The Politics of Recognition ", Princeton, N.J., Princeton University Press, 1992, p. 58. 
pluraliste n'arriveront pas à se mettre d'accord sur un catalogue de ces biens. Mais supposons que la disponibilité d'au moins une partie de ces biens ne puisse ètre assurée par le fonctionnement normal du marché. Est-ce que ce désaccord empècherait l'État de s'engager dans leur protection?

La conception perfectionniste modeste répond par la négative à cette question. Si nous sommes intéressês à réaliser la bonne conception du bien et pas seulement la conception du bien que nous avons librement choisie, alors nous aurons toujours des raisons pour préférer un contexte de choix plus riche à un contexte de choix plus pauvre. Et nous aurons même des raisons pour accepter que le soutien accordé à certains biens spécifiques rend certaines conceptions du bien (plus ou moins "déterminées") plus réalisables que d'autres, dès lors que l'absence de tout soutien de cette sorte rendrait notre recherche du bien encore plus difficile. L'important est que : i) la procédure d'identification des biens à protéger ne soit pas fondée sur des jugements sur la valeur intrinsèque des différentes conceptions du bien présentes dans la société ou des individus euxmèmes, et ii) que le catalogue des biens à protéger soit considéré à chaque instant comme étant provisoire et révisable.

Cette argumentation ne suffit évidemment pas à légitimer le point de vue perfectionniste modeste. Pour $y$ arriver il faudrait encore montrer : i) que la protection de la richesse du contexte de choix n'est possible que moyennant l'intervention de l'État, et ii) que cette intervention n'est possible que si l'État se rend sensible à certains jugements de valeur à propos de ce qui mérite d'être protégé. Je ne discuterai pas ici ces deux affirmations, car je n'essaie pas de vérifier si nous avons effectivement des raisons pour préférer le perfectionnisme modeste à la conception anti-perfectionniste de la neutralité. Ce qui m'intéresse c'est plutōt de montrer que ce choix existe en tant que possibilité conceptuelle, c'est-à-dire que la conception perfectionniste modeste est aussi une conception plausible de la neutralité de l'État. Je me limiterai donc à ajouter trois précisions.

D'abord il faut souligner que le perfectionnisme modeste n'exige pas que les biens partagés soient des biens "intersectionnels ", c'està-dire des biens dont la valeur est reconnue par toutes les conceptions du bien présentes dans la société. Car un bien " intersectionnel " est un bien dont la valeur n'est incontestée que pour le moment. Il suffirait donc qu'un individu le mette en question pour qu'il cesse d'être un bien digne d'être soutenu par l'État. En fait, il n'est pas nécessaire que les biens partagés soient unanimement acceptés. En tentant de les identifier nous ne visons pas à répondre à la question : "quels sont les biens recherchês par tous les membres de notre société ? ". mais plutôt à la question : "quels sont les biens susceptibles d'enrichir notre contexte de choix, étant données nos intuitions les plus solides à propos de la vie politique, nos traditions publiques les plus stables et les particularités de notre histoire et de notre culture?".

Cette dernière question recevra des réponses différentes d'un individu à l'autre, mais cela n'empèche pas qu'on puisse s'accorder sur la valeur d'au moins certains biens. Par exemple, Joseph Raz a montré que, dans le cadre d'une société ayant fait un choix en faveur de la démocratie constitutionnelle, les individus auront aussi des 
raisons pour préférer une discussion publique plus rationnelle et mieux informée. Or, une presse qui ne soit pas seulement libre mais aussi influente et puissante favorise ce type de discussion. L'État peut donc lui accorder des droits unilatéraux allant au delà du simple respect de la liberté d'expression (protection des sources, accès préférentiel aux tribunaux, etc.), non pas en raison d'une exigence de justice mais dans le but de soutenir un bien qui est reconnu comme important et partage $\mathrm{e}^{55}$. Ce raisonnement ne prétend pas que la totalité des citoyens considérera la bonne qualité de la discussion publique comme un bien partagé. Il suggère plutôt que la protection de ce bien est compatible avec (et même exigée par) notre choix collectif en faveur de la démocratie constitutionnelle. S'il y a donc une majorité de citoyens favorable à ce choix, on ne pourra pas s'opposer à la protection de ce bien par un recours au principe de neutralité.

Ensuite, les biens partagés ne sont pas non plus ceux qui font l'objet d'un recoupement entre toutes les conceptions raisonnables du bien individuel présentes dans la société. Cette idée a été récemment proposée par William Galston ${ }^{56}$, mais elle reste fort problematique. Certes, certains biens font normalement partie des conceptions du bien développées par des individus dès lors qu'ils se conçoivent comme des agents moraux rationnels et raisonnables. Mais tout le problème réside dans l'ambiguité du mot " normalement ", car les critères de délimitation sont difficiles à établir : quelles seront, en effet, les conceptions du bien qui seront prises en compte dans le consensus raisonnable et quelles seront les conceptions qui seront rejetées?

Enfin, le perfectionnisme modeste ne propose pas non plus une restriction purement instrumentale à l'anti-perfectionnisme, comme celle qui est proposée par une partie de la tradition républicaine. En effet, plusieurs auteurs ont soutenu, au moins depuis Machiavel, que l'État doit encourager certaines valeurs (par exemple, la participation dans la vie publique) non pas pour des raisons perfectionnistes, mais parce que cela est une condition de la stabilité politique. Le problème de savoir si la participation à la vie publique est une composante essentielle de la vie bonne n'est pas pertinent ici. Leur position est qu'il faut admettre que sans un engagement des citoyens dans la vie publique, le régime républicain n'arrivera pas à se maintenir ${ }^{57}$. Le perfectionnisme modeste, par contre, ne vise pas en premier lieu les conditions de la stabilité politique (quoique ce problème reste important) mais les conditions de possibilité d'une recherche indépendante du bien menée dans le cadre d'une vie sociale

55. J. Raz. The Morality of Freedom, p. 198 ss et p. 253.

56. Galston a proposé un catalogue de biens issus d'une " common experience of the bad ". Il inclut la préservation de la vie, le développement normal de nos capacités de base, la réalisation de nos intérêts et projets, l'exercice de la liberté de choix et de la rationalité, l'appartenance à un tissu de rapports sociaux et les expériences de satisfaction subjective. Voir son Liberal Purposes. Goods, Virtues, and Diversity in the Liberal State, Cambridge, Cambridge University Press, p. 168 ss.

57. Quentin Skinner. "On Justice, the Common Good and the Priority of Liberty ". Dans Chantal Mouffe (ed.), Dimensions of Radical Democracy. London \& New York, Verso, 1992, p. 219-221. 
caractérisée par le fait du pluralisme et la rareté modérée des ressources. L'accord constitutionnel (c'est-à-dire, l'accord qui rend possible la cœxistence politique) est perçu comme un accord visant à rendre possible une telle vie pour tous les membres de la société.

La conception perfectionniste modeste de la neutralité est donc plus faible que la conception anti-perfectionniste, mais elle reste toujours une conception de la neutralité. Elle se distingue de l'antiperfectionnisme, mais elle se distingue encore plus radicalement du perfectionnisme tout court. Cela veut dire qu'elle rejette trois thèses qui ont été historiquement associées à ce point de vue. D'abord, elle rejette la thèse affirmant la supériorité intrinsèque de certains individus par rapport aux autres. Ensuite elle rejette la thèse affirmant la supériorité intrinsèque de certaines conceptions du bien (et des formes de vie qui leurs sont associées) par rapport aux autres $^{58}$. Enfin, elle rejette la thèse affirmant qu'il y a un bien commun (ou un ensemble de biens) partagé par tous les membres de la sociêté.

La conception perfectionniste modeste affirme plutôt qu'il y a des biens qui peuvent ètre reconnus comme partagés par les membres d'une société pluraliste, et elle ajoute que ces biens peuvent étre soutenus et protégés par un État neutre pourvu que cela se fasse par des procédures compatibles avec l'égal respect des individus. On est donc toujours en présence d'une conception de la neutralité de l'État : les actions de celui-ci doivent être justifiées sans faire appel à des jugements intrinsèques sur les conceptions non-minimales du bien choisies par les individus ou sur les individus eux-mêmes. Et. comme même les anti-perfectionnistes le reconnaissent, "dans la mesure où un gouvernement conforme ses décisions à cette contrainte, il agit de façon neutre ${ }^{59}$.

\section{Un concept, de ux conceptions}

Selon nos définitions de départ, tant l'anti-perfectionniste que le perfectionniste modeste soutiennent un principe de neutralité des justifications. Le premier affirme que l'action de l'État doit ètre aveugle à tout jugement de valeur, c'est-à-dire qu'étant donnés deux groupes d'individus $A$ et $B$, l'État ne peut agir d'une façon différente par rapport aux deux collectifs en faisant appel à des jugements de valeur sur les conceptions du bien défendues dans chaque cas, ni sur les biens particuliers recherchés par chaque groupe, ni sur les qualités des membres qui appartiennent à l'un et l'autre ensemble. Cette formulation impose des limitations aux justifications de l'action de l'État, mais elle ne dit pas que l'État ne peut pas agir d'une façon telle que les conséquences de son action soient differentes pour les membres des deux groupes. En revanche, le deuxième affirme que,

58. Comme Vinit Haksar a argumenté, " one can quite consistently believe that some forms of human life are intrinsically superior to other forms of human life without believing that some human beings are intrinsically superior to other human beings "(Equality. Liberty and Perfectionism Oxford, Oxford University Press, 1979, p. 2). Ce type d'affirmation peut donc faire partie des convictions personnelles légitimes des citoyens d'une démocratie constitutionnelle. Mais le principe de neutralité interdit à l'État de se prononcer sur ce sujet.

59. C. Larmore, Pattems of Moral Complexity, p. 44. 
dans la justification de ses actions, l'État ne peut pas aller au delà des accords publics à propos des biens qui sont considérés comme étant partagés. Cela implique que, étant donnés deux groupes $A$ et $B$, l'État ne peut agir de façon différente envers les membres de ces groupes que s'il peut faire appel à des considérations de justice ou à la protection des biens ou des pratiques reconnus comme partagés. Cette définition ne dit pas non plus que l'action de l'État ne peut pas avoir de conséquences différentes pour les membres des deux groupes.

Les deux conceptions se différencient, néanmoins, en regard de la distinction entre neutralité envers les individus et neutralité envers les idéaux. Dans les deux cas la neutralité envers les individus est prioritaire, mais la neutralité envers les conceptions du bien est plus radicale dans le cas de l'anti-perfectionnisme que dans le cas du perfectionnisme modeste. En effet, pour le défenseur du point de vue anti-perfectionniste, les actions de l'Etat ne pourront avoir de conséquences différentes sur des idéaux différents que si elles sont fondées sur des arguments de justice ou d'efficience. Le défenseur du point de vue perfectionniste modeste admet, quant à lui, que l'État ne peut pas se substituer au jugement des individus à propos des conceptions du bien, mais il affirme que les différences au niveau des conséquences peuvent aussi être justifiées en faisant appel à la protection de certains biens qui ont été reconnus comme partagés par les individus.

\section{Conclusion}

Cette discussion m'a permis de trouver quelques critères de distinction entre un État neutre et un État qui ne l'est pas. D'après mes conclusions, en effet, un État n'est neutre que si : i) il ne prétend exprimer aucune conception "profonde "sur notre vie morale mais il reste au niveau de l'argumentation politique ; ii) au lieu de viser une neutralité des conséquences, il essaie de justifier ses actions d'une façon acceptable pour tous les membres de la société ; iii) il reconnait la priorité du respect des individus par rapport au respect des idéaux. Par contre, nous avons des raisons pour rejeter un quatrième critère possible et d'affirmer que : iv) un État ne doit pas ètre minimal pour revendiquer sa neutralité, pourvu qu'il soit capable de respecter les trois critères précédents.

Cette discussion m'a permis aussi d'identifier deux conceptions de la neutralité qui en principe respectent l'interdiction adressée à l'État de s'engager dans des évaluations intrinsèques sur les individus ou sur les conceptions du bien choisies par eux. Je n'ai pas traité ici les questions de savoir si : i) l'une de ces conceptions est préférable à l'autre, et ii) si, même dans ce cas, la différence entre l'une et l'autre aura des conséquences importantes dans la pratique. Je suggère plutôt que toutes les deux peuvent être comprises comme des interprétations du concept de neutralité, de sorte que toutes les deux partagent une certaine confiance dans les procédures nonétatiques pour créer les conditions favorables à notre vie morale et au développement culturel, ainsi qu'une certaine méfiance à l'égard d'un 
engagement de l'État dans des évaluations sur le bien ${ }^{60}$. Mais l'une se différencie de l'autre selon la portée qu'elles reconnaissent à cette confiance et à cette méfiance.

Je voudrais finir en formulant une hypothèse que je ne saurais pas défendre dans les limites de cet article: si le concept de neutralité est devenu si problématique aux yeux des philosophes et mème aux yeux des acteurs sociaux (pensons, par exemple, au cas de l'enseignement) cela est dū au fait que nos théories sur la neutralitê ont été bâties sur une base anti-perfectionniste, tandis que les pratiques que nous demandons aux responsables publics sont fondées sur une sensibilité perfectionniste modeste plus au moins explicite. Ce n'est que si nous prenons conscience de ce décalage que nous pourrons maitriser les difficultés auxquelles nous nous heurtons quand nous essayons de mettre en œuvre cet impératif ${ }^{61}$.

\section{CLAEH}

Université Catholique de l'Uruguay (Montevideo)

60. W. Kymlicka, Contemporary Political Philosophy, p. 222.

61. Je remercie André Berten, Monique Canto-Sperber, Hervé Pourtois et Philippe Van Parijs pour leurs commentaires lors de la préparation de cet article. 